TRANSACTIONS OF THE

AMERICAN MATHEMATICAL SOCIETY

Volume 354, Number 11, Pages 4571-4583

S 0002-9947(02)03049-0

Article electronically published on July 2, 2002

\title{
EMERGENCE OF THE WITT GROUP IN THE CELLULAR LATTICE OF RATIONAL SPACES
}

\author{
KATHRYN HESS AND PAUL-EUGÈNE PARENT
}

\begin{abstract}
We give an embedding of a quotient of the Witt semigroup into the lattice of rational cellular classes represented by formal 2-cones between $S^{2 n}$ and the two-cell complex $X_{n}=S^{2 n} \cup_{[\iota, \iota]} e^{4 n}(n \geq 1)$.
\end{abstract}

\section{INTRODUCTION}

It is now a well-established fact in unstable homotopy theory that the structure of the Dror Farjoun (cellular) lattice $\left(T o p_{*}, \ll\right)$ 8] defined on the category of pointed spaces having the homotopy type of a CW-complex is highly nontrivial. At the time this paper is being written its full classification is still intractable, to the best of the authors' knowledge. Just recently the partial order was in fact shown to be a (complete) lattice [7. The dream of course would be to classify it, as Hopkins and Smith [12] did in the case of the Bousfield lattice [3] of stable $p$-torsion finite complexes.

In a first attempt to tackle this classification problem, Chachólski, Stanley and the second author came up with a criterion to determine when $A \ll B$ rationally [6]. This criterion can be extended to the case of spaces that are Bousfield equivalent to $S^{n}$, i.e., $(n-1)$-connected spaces $A$ such that $\operatorname{map}_{*}\left(S^{n}, X\right) \simeq *$ whenever $\operatorname{map}_{*}(A, X) \simeq *$. We immediately deduce the following strict building relation:

$$
S^{2} \ll \mathbb{C} P^{2} \ll \mathbb{C} P^{3} \ll \ldots \ll \mathbb{C} P^{\infty} .
$$

Afterwards, Félix constructed yet another such example over the rationals, i.e.,

$$
S^{2} \ll \ldots \ll \underbrace{\mathbb{C} P^{2} \# \ldots \# \mathbb{C} P^{2}}_{n \text { times }} \ll \ldots \ll \mathbb{C} P^{2} \# \mathbb{C} P^{2} \ll \mathbb{C} P^{2} .
$$

Furthermore, using the Chachólski-Parent-Stanley criterion together with results concerning inert cell attachments [10, the first author produced an infinite family of cellularly incomparable spaces, which all have the same rational homotopy Lie algebra and the same rational homology coalgebra [11. Even rationally, therefore, the structure of the cellular lattice is remarkable.

In this paper we continue the quest for the holy grail of complete classification of the rational cellular lattice. We consider cellular classes generated by $(2 k-1)$ connected spaces $X$ of dimension at most $6 k-1(k \geq 1)$ such that $\pi_{2 k}(X) \otimes \mathbb{Q} \neq 0$. Moreover, we ask that these spaces be rationally formal. In these cases, for degree reasons, the only relevant cells are in dimensions $2 k$ and $4 k$. The crucial remark is

Received by the editors November 6, 2001 and, in revised form, March 19, 2002.

2000 Mathematics Subject Classification. Primary 11E04, 55P60, 55P62.

Key words and phrases. Cellular space, quadratic form, Witt group, Quillen model. 
that all these cellular classes are contained between $S^{2 k}$ and the two-cell complex $X_{k}=S^{2 k} \cup_{[\iota, \iota]} e^{4 k}(k \geq 1)$ (Proposition [3). Let $\mathbb{M}$ be the set of cellular classes generated by such spaces with at most one cell in dimension $4 k$. In Theorem 7 , we show that $\mathbb{M}$ is in one-to-one correspondence with a certain quotient $\overline{W(\mathbb{Q})}$ of the Witt semigroup $W(\mathbb{Q})$, where the operation considered is the Kronecker product of quadratic forms. The quadratic forms within this quotient have been classified in the work of Lewis and Tignol 13, where they generalize the classical invariants, i.e., Clifford algebra, signature, and discriminant, associated to isometry classes of quadratic forms (see the Appendix).

Thanks to the isomorphism between $\mathbb{M}$ and $\overline{W(\mathbb{Q})}$, there is a partial order on $\overline{W(\mathbb{Q})}$, corresponding to the building relation on cellular classes. We believe this partial order to have been unknown previously and intend to study the implications of its existence for the classification of quadratic forms.

Finally, we exhibit an action of $\mathbb{M}$ on the set of cellular classes generated by these 2-cones where the restriction on the number of $4 k$-cells is lifted. To the best of the authors' knowledge, this is the first time that such algebraic operations have been defined on a subset of the rational lattice.

\section{NOTATION AND BACKGROUND}

Throughout this paper all spaces are rationalizations of simply-connected spaces having the homotopy type of a CW-complex.

2.1. Rational homotopy theory and Quillen models. Rational homotopy theory has its roots in the work of Quillen [15] and Sullivan [17. For a complete overview of these techniques, we refer the reader to [9]. We recall here some of the highlights, in particular, the equivalence of categories between the homotopy category of simply-connected rational spaces and the homotopy category of connected differential graded rational Lie algebras.

Recall that a graded Lie algebra over the field of rational numbers $\mathbb{Q}$ consists of a positively graded $\mathbb{Q}$-vector space $L$, together with a bilinear product called the Lie bracket that we denote $[-,-]$, such that

$$
[x, y]=-(-1)^{|x||y|}[y, x]
$$

and

$$
[x,[y, z]]=[[x, y], z]+(-1)^{|x||y|}[y,[x, z]]
$$

for all homogeneous $x, y, z \in L$, where $|\alpha|$ refers to the degree of a homogeneous element $\alpha \in L$. This last identity is known as the Jacobi identity and simply expresses the fact that the adjoint representation acts as a derivation with respect to the bracket.

If a graded Lie algebra $L$ is endowed with a derivation $\partial$ of degree -1 such that $\partial^{2}=0$, we call $(L, \partial)$ a differential graded Lie algebra, abbreviated $d g L$, and $\partial$ is its differential.

Let $V$ be a positively-graded $\mathbb{Q}$-vector space, and let $T V$ denote the tensor algebra on $V$. When endowed with the commutator bracket, $T V$ is a graded Lie algebra. The free Lie algebra on $V$, denoted $\mathbb{L} V$, is the smallest sub Lie algebra of $T V$ containing $V$. An element in $\mathbb{L} V$ has bracket length $k$ if it is a linear combination of iterated brackets of $k$ elements of $V$, i.e., if it belongs to the intersection $\mathbb{L} V \cap T^{k} V$, where $T^{k} V$ denotes the subspace of $T V$ generated by the words of tensor length 
$k$. The subspace of elements of bracket length $k$ is denoted $\mathbb{L}^{k} V$. Note that any graded linear map of degree 0 from $V$ into a graded Lie algebra $L$ can be extended uniquely to a Lie algebra map from $\mathbb{L} V$ into $L$. We say that a dgL is free if the underlying graded Lie algebra is free. The differential $\partial$ of a free $\operatorname{dgL}(\mathbb{L} V, \partial)$ can be decomposed as $\partial=\partial_{1}+\partial_{2}+\partial_{3}+\ldots$, where $\partial_{k}(V) \subset \mathbb{L}^{k} V$. The summand $\partial_{2}$ is called the quadratic part of the differential $\partial$.

Let $X$ be a simply-connected space. The graded vector space $\pi_{*}(\Omega X) \otimes \mathbb{Q}$ admits a natural graded Lie algebra structure, where the bracket is given by the Samelson product. Together with the Samelson bracket, $\pi_{*}(\Omega X) \otimes \mathbb{Q}$ is called the rational homotopy Lie algebra of $X$, denoted $\mathcal{L}(X)$ in this article.

If $X$ is simply-connected, then there exists a free $\operatorname{dgL} \mathcal{M}(X)=(\mathbb{L} V, \partial)$, unique up to isomorphism, called the Quillen (or Lie) model of $X$. It completely characterizes the rational homotopy type of the space $X$ and has, in particular, the following properties:

- $H_{*}(\mathcal{M}(X)) \cong \mathcal{L}(X)$ as graded Lie algebras;

- $s V \cong \widetilde{H}_{*}(X ; \mathbb{Q})$ as graded vector spaces, where $(s V)_{n}=V_{n-1}$ for all $n>0$;

- $\partial_{1}=0 ;$ and

- $\partial_{2}$ is obtained via desuspension from the coproduct on $H_{*}(X ; \mathbb{Q})$.

Moreover, geometrically this model corresponds to a cell decomposition where the cells are represented by the generators, and the attaching maps are encoded into the differential. This model is intimately linked to the Adams-Hilton model [1], as shown by Anick in [2]. We will thus refer to an inclusion of the type $\mathbb{L}\left(V_{<n}\right) \hookrightarrow \mathbb{L}(V)$ as the inclusion of the $n^{\text {th }}$-skeleton of $X$ into $X$.

Dually (in the sense of Eckmann-Hilton), when the rational homology of $X$ is of finite type, the essential property of the Quillen model of $X$ is that there is a quasi-isomorphism of commutative cochain algebras from the Cartan-ChevalleyEilenberg construction on $\mathcal{M}(X)$ to the de Rham algebra of piecewise-linear forms on the singular set of $X$.

Finally, the Quillen model is a natural construction, so that a continuous map between two simply-connected spaces induces a dgL-map between their respective Quillen models.

2.2. Cellular spaces. In [8] Dror Farjoun made the following definition. A full subcategory of pointed spaces, $\mathcal{C} \subset$ Top* ${ }^{*}$, is called a closed class if it is closed under weak equivalences and arbitrary pointed homotopy colimits. We refer the reader to the work of Bousfield and Kan [4] and to Dror Farjoun's book [8] for the definitions and constructions associated with the notion of (pointed) homotopy (co)limits. A result of Chachólski in [5] shows that a class $\mathcal{C}$ of spaces is a closed class if and only if

- if $X \in \mathcal{C}$ and $Y \simeq X$, then $Y \in \mathcal{C}$; and

- if $X_{i} \in \mathcal{C}$, where $i$ belongs to some small category $I$, then

$-\operatorname{hocolim}_{*}\left(X_{1} \rightarrow X_{2} \rightarrow \cdots\right) \in \mathcal{C}$,

$-\operatorname{hocolim}_{*}\left(X_{1} \leftarrow X_{2} \rightarrow X_{3}\right) \in \mathcal{C}$, and

- $\bigvee_{i \in I} X_{i} \in \mathcal{C}$.

An important example of such a class is $C(A)$, the smallest closed class containing the space $A$ (e.g., $C\left(S^{n}\right)$ is the class of all $(n-1)$-connected spaces). Thus we say that $B$ is $A$-cellular, or that $A$ builds $B$, which we denote $A \ll B$, if $B \in C(A)$. Two spaces $A$ and $B$ are cellularly equivalent, denoted $A \tilde{c} B$, if $A \ll B$ and $B \ll A$. 
We say that two Quillen models are cellularly equivalent if their corresponding rational spaces are equivalent.

The following theorem, due to Chachólski, Stanley, and the second author, is the foundation of the research presented in this article.

Theorem 1 ([6]). Let $X$ and $A$ be two $(n-1)$-connected $(n>1)$ rational spaces such that $\pi_{n}(X)$ and $\pi_{n}(A)$ are nontrivial. Then $A \ll X$ if and only if there exists a continuous map $f: \vee_{i \in J} A \rightarrow X$ for some index set $J$, such that $\pi_{n}(f)$ is surjective.

This result, as mentioned by the first author in [11, has the following algebraic translation. Let $X$ and $A$ be as in the previous theorem. Then $A \ll X$ if and only if there is a dgL morphism $\phi: \coprod_{i \in J} \mathcal{M}(A) \rightarrow \mathcal{M}(X)$ inducing a homology surjection in degree $n-1$, where $J$ is an arbitrary index set, and the coproduct is taken in the dgL category. It is thus obvious that any nontrivial $n^{\text {th }}$-skeleton of $X$ builds $X$.

Proposition 2. Let $X$ be an $(n-1)$-connected space such that $\pi_{n}(X) \neq 0$. If the differential $\partial$ of its Quillen model $(\mathbb{L}(V), \partial)$ is such that $\partial_{2}=0$, then $X \tilde{c} S^{n}$.

Proof. This is a consequence of the Jacobi identity, which implies that all triple brackets of the type $[x,[x, x]]$ are zero. Let $(\mathbb{L}(\omega), 0)$ be the Quillen model of $S^{n}$ where $|\omega|=n-1$. Choose a basis $\left\{x_{1}, \ldots, x_{k}\right\}$ for $V_{n-1}$, and define a dgL morphism $\left(\mathbb{L}\left(V_{n-1} \oplus V_{\geq n}\right), \partial\right) \rightarrow(\mathbb{L}(\omega), 0)$ by sending $x_{1}$ to $\omega$, all $x_{i}$ to zero for $i>1$, and $V_{\geq n}$ also to zero. This is indeed a dgL morphism since any bracket of length at least three must contain a generator different from $x_{1}$. Clearly this morphism induces a homology surjection in degree $n-1$.

Evidently the quadratic part of the differential plays a crucial role in furnishing examples of spaces cellularly distinct from the spheres. In a first attempt to classify the rational cellular lattice, we restrict our analysis primarily to models such that $\partial=\partial_{2}$, i.e., rational formal spaces.

Finally, recall a result from [6] (for an algebraic proof see [11]) which states that if $X$ is a $(2 k)$-connected rational space such that $\pi_{2 k+1}(X) \neq 0$, then $X \tilde{c} S^{2 k+1}$. Thus the relevant part of the analysis is done for $(2 k-1)$-connected spaces $X$ such that $\pi_{2 k}(X) \neq 0$.

2.3. Quadratic forms. We refer the reader to [16 for the proof of the classical results on quadratic forms. We recall here the relevant results for this paper.

All quadratic forms that we consider are regular rational forms of finite dimension. Let $Q$ and $Q^{\prime}$ be two forms of the same dimension $n$. They are said to be isometric, denoted $Q \simeq Q^{\prime}$, if there exists an invertible $n \times n$ matrix $C$ such that $A_{Q}=C A_{Q^{\prime}} C^{\dagger}$, where $A_{Q}$ and $A_{Q^{\prime}}$ are the matrices associated to $Q$ and $Q^{\prime}$, respectively. They are similar, denoted $Q \sim Q^{\prime}$, if there exists a nonzero rational number $r$ such that $r Q \simeq Q^{\prime}$.

A form $Q$ is said to be isotropic if there exists a nontrivial vector $v$ such that $Q(v)=0$. Otherwise it is said to be anisotropic. Note that the empty form $\phi=0$ of dimension 0 is counted as being regular and anisotropic.

A classical result asserts that over any field of characteristic different from two, any $n$-dimensional form is isometric to a diagonal form. We use the notation $\left\langle\alpha_{1}, \alpha_{2}, \ldots, \alpha_{n}\right\rangle$ to denote a diagonal form, where the $\alpha_{i}$ are the diagonal entries. 
We have two operations defined up to isometry, i.e., given $Q \simeq\left\langle\alpha_{1}, \alpha_{2}, \ldots, \alpha_{n}\right\rangle$ and $Q^{\prime} \simeq\left\langle\beta_{1}, \beta_{2}, \ldots, \beta_{m}\right\rangle$ one has the sum and the product

$$
Q \perp Q^{\prime} \simeq\left\langle\alpha_{1}, \ldots, \alpha_{n}, \beta_{1}, \ldots, \beta_{m}\right\rangle \quad \text { and } \quad Q \otimes Q^{\prime} \simeq \perp_{i=1}^{n}\left\langle\alpha_{i} \beta_{1}, \ldots, \alpha_{i} \beta_{m}\right\rangle .
$$

Note that only the product is well defined up to similarity.

Example. Let $Q=\langle 1\rangle$ and $Q^{\prime}=\langle-1\rangle$. We have $Q \sim Q^{\prime}$ (clearly $Q \not Q^{\prime}$ ). Then $Q \perp Q \nsim Q \perp Q^{\prime}$ since $Q \perp Q$ is anisotropic while $Q \perp Q^{\prime}$ is isotropic.

In general, the sum or the product of two anisotropic forms is not anisotropic as the following examples show. Consider $Q \simeq\langle 1,1\rangle$ and $Q^{\prime} \simeq\langle-1,2\rangle$. They are both anisotropic, but their sum $\langle 1,1,-1,2\rangle$ and their product $\langle-1,2,-1,2\rangle$ are not. A result known as the Witt Decomposition Theorem says that any form $\phi$ is isometric to

$$
\underbrace{\langle 1,-1\rangle \perp \ldots \perp\langle 1,-1\rangle}_{i} \perp \phi_{o}
$$

where $\phi_{o}$ is anisotropic and uniquely determined by $\phi$ up to isometry. In the examples above, $\left(Q \perp Q^{\prime}\right)_{o}$ is isometric to $\langle 1,2\rangle$, while $\left(Q \otimes Q^{\prime}\right)_{o}$ is isometric to the empty quadratic form. We call the association $\phi \mapsto \phi_{o}$ the reduction of $\phi$.

The set of isometry classes of anisotropic forms $W(\mathbb{Q})$ equipped with the two operations $\perp$ and $\otimes$ is known as the Witt ring where the appropriate reductions are applied. We denote the set of similarity classes of anisotropic forms by $\overline{W(\mathbb{Q})}$. Thus $(\overline{W(\mathbb{Q})}, \otimes)$ becomes a semigroup with unity $\langle 1\rangle$, the unique 1-dimensional similarity class. Moreover, we have a natural semigroup epimorphism

$$
W(\mathbb{Q}) \longrightarrow \overline{W(\mathbb{Q})} .
$$

\section{MAIN RESUlts}

Let $k$ be a positive integer. Let $M=\left(\mathbb{L}(X \oplus W \oplus Y \oplus Z), \partial=\partial_{2}\right)$ be a Quillen model, where $X, W, Y$, and $Z$ are graded vector spaces such that

- $X$ is of dimension $n>0$ and is concentrated in degree $2 k-1$;

- $W$ is nontrivial in at most degrees $2 k$ through $4 k-2$;

- $Y$ is of dimension $m$ and is concentrated in degree $4 k-1$; and

- $Z$ is nontrivial in at most degrees $4 k$ through $6 k-2$.

In other words, $M$ is the Quillen model of a $(2 k-1)$-connected $\mathrm{CW}$-complex of dimension at most $6 k-1$, with $n$ cells of dimension $2 k$ and $m$ cells of dimension $4 k$. For degree reasons $\partial(X \oplus W)=0$, while $\partial(Y)$ corresponds to $m$ choices of $n$-dimensional quadratic forms on $X$. Indeed, given bases $\left\{x_{1}, \ldots, x_{n}\right\}$ and $\left\{y_{1}, \ldots, y_{m}\right\}$ for $X$ and $Y$, respectively, $\partial\left(y_{i}\right)$ is a rational homogeneous polynomial of degree 2 in the variables $x_{j}$, i.e.,

$$
\partial\left(y_{i}\right)=\sum_{j=1}^{n} \alpha_{j}\left[x_{j}, x_{j}\right]+\sum_{l<j} \beta_{l, j}\left[x_{l}, x_{j}\right] \quad \text { with } \quad \alpha_{j}, \beta_{l, j} \in \mathbb{Q} .
$$

Notice that $\left[x_{l}, x_{j}\right]=\left[x_{j}, x_{l}\right]$ since the degree of the $x_{j}$ is odd.

Moreover, $\partial(Z) \subset \mathbb{L}^{2}(X \oplus W)$ with at least one nontrivial $w \in W$ in each bracket. Note that if $Z_{6 k-2}=0$, then the formality condition is automatically satisfied, i.e., $\partial=\partial_{2}$. Clearly, from the inclusion and the projection we get

$$
M \tilde{c}\left(\mathbb{L}(X \oplus Y), \partial=\partial_{2}\right) .
$$


Moreover, the following relations hold among these models.

\section{Proposition 3.}

$$
\mathcal{M}\left(S^{2 k}\right) \cong(\mathbb{L}(x), 0) \ll\left(\mathbb{L}(X \oplus Y), \partial=\partial_{2}\right) \ll(\mathbb{L}(x, y), \partial(y)=[x, x]) \cong \mathcal{M}\left(X_{k}\right) .
$$

Proof. The only thing to show is the right-hand building relation. Choose bases $\left\{x_{1}, \ldots, x_{n}\right\}$, and $\left\{y_{1}, \ldots, y_{m}\right\}$ for $X$ and $Y$, respectively. Denote by $Q_{j}$ the $j^{\text {th }}$ quadratic form $\partial\left(y_{j}\right)$ on $X(1 \leq j \leq m)$. Define a Lie algebra map

$$
\phi:\left(\mathbb{L}(X \oplus Y), \partial=\partial_{2}\right) \rightarrow(\mathbb{L}(x, y), \partial(y)=[x, x])
$$

by sending $x_{i}$ to $x(1 \leq i \leq n)$ and $y_{j}$ to $Q_{j}(v) y$, where $v$ is the $n$-tuple $(1, \ldots, 1)$. A simple computation shows that $\phi$ is a dgL-morphism that induces a homology surjection in degree $2 k-1$.

As a first step, we consider spaces having $M_{n}=\left(\mathbb{L}(X \oplus Y), \partial=\partial_{2}\right)$ as Lie models where $X$ and $Y=\mathbb{Q} y$ are respectively $n$ - and 1-dimensional. We will denote by $Q_{M}$ the quadratic form $\partial y$ on $X$. Thus we can speak of an isotropic or anisotropic model $M_{n}$ if its associated quadratic form has the respective characteristic. We denote by $\mathbb{M}$ the set of closed classes generated by the anisotropic models. The fact that the set of quadratic forms is partitioned in two, i.e., the isotropic forms versus the anisotropic ones, together with the following proposition and the initial remarks of this section show that this definition is equivalent to the one given in the introduction.

Proposition 4. A model $M_{n}$ is isotropic if and only if $M_{n} \tilde{c} S^{2 k}$.

Proof. Let $M_{n}=\left(\mathbb{L}\left(x_{1}, \ldots, x_{n}, y\right), \partial y=Q_{M}\right)$. If $Q_{M}$ is isotropic, then there is a nontrivial $V=\left(v_{1}, \ldots, v_{n}\right)$ such that $Q_{M}(V)=0$. But then, it is easy to check that the map $\phi: M_{n} \rightarrow(\mathbb{L}(\omega), 0)$ defined by $\phi\left(x_{i}\right)=v_{i} \omega$ for $1 \leq i \leq n$, and $\phi(y)=0$ is a dgL-morphism that is an $H_{2 k-1}$-surjection. Conversely, if $M_{n} \tilde{c} S^{2 k}$, then there is a dgL-morphism $\phi: M_{n} \rightarrow(\mathbb{L}(\omega), 0)$ that is an $H_{2 k-1}$-surjection. Let $V$ be the $(1 \times n)$-matrix associated to the linear map $\phi_{2 k-1}$. Again, the requirement that $\phi$ be a dgL-morphism implies that $Q_{M}(V)=0$.

Lemma 5. If a morphism $\phi: M_{n} \rightarrow M_{m}^{\prime}$ between anisotropic models is such that $n \geq m$ is nontrivial, then it is an $H_{2 k-1}$-surjection. Moreover, there are no nontrivial maps when $n<m$.

Proof. Let $M_{n}=\left(\mathbb{L}\left(x_{1}, \ldots, x_{n}, y\right), \partial y=Q_{M}\right)$ and $M_{m}^{\prime}=\left(\mathbb{L}\left(x_{1}^{\prime}, \ldots, x_{m}^{\prime}, y^{\prime}\right), \partial^{\prime} y^{\prime}=\right.$ $\left.Q_{M^{\prime}}\right)$. Let $C$ be the $(m \times n)$-matrix representing the linear map $\phi_{2 k-1}$ with respect to the bases $\left\{x_{1}, \ldots, x_{n}\right\}$ and $\left\{x_{1}^{\prime}, \ldots, x_{m}^{\prime}\right\}$, and let $\alpha \in \mathbb{Q}$ be the scalar such that $\phi(y)=\alpha \cdot y^{\prime}$. A simple but tedious computation shows that $\alpha \cdot A^{\prime}=C A C^{\dagger}$, where $A$ and $A^{\prime}$ are the matrix representations of $Q_{M}$ and $Q_{M^{\prime}}$, respectively. If $\alpha=0$, then each row vector of $C$ is a zero of $Q_{M}$, and since $\phi$ is not trivial, there must be one such row that is nonzero. This would contradict the fact that $Q_{M}$ is anisotropic, and hence $\alpha \neq 0$. Now, if $\phi_{2 k-1}$ is not onto, then $r k(C)<m$, i.e., there is a nontrivial solution to the homogeneous system $v C=0$. The result follows since nontrivial solutions would imply $Q_{M^{\prime}}$ isotropic, a contradiction. Finally, if $n<m$, then there are no nontrivial maps since the homogeneous system $v C=0$ always has a nontrivial solution. 
Proposition 6. Two anisotropic models $M_{n}$ and $M_{n}^{\prime}$ generate the same cellular class, i.e., $M_{n} \tilde{c} M_{n}^{\prime}$, if and only if their associated quadratic forms are similar, i.e., $Q_{M} \sim Q_{M^{\prime}}$.

Proof. Let $M_{n}=\left(\mathbb{L}\left(x_{1}, \ldots, x_{n}, y\right), \partial y=Q_{M}\right)$ and $M_{n}^{\prime}=\left(\mathbb{L}\left(x_{1}, \ldots, x_{n}, y\right), \partial^{\prime} y=\right.$ $Q_{M^{\prime}}$ ). Let $A$ and $A^{\prime}$ be the matrix representations of $Q_{M}$ and $Q_{M^{\prime}}$, respectively. If $M_{n} \tilde{c} M_{n}^{\prime}$, then, by Lemma 5 there is a dgL-morphism $\phi: M_{n} \rightarrow M_{n}^{\prime}$ that is an $H_{2 k-1}$-surjection. Let $C$ be the matrix representing the linear map $\phi_{2 k-1}$ with respect to the basis $\left\{x_{1}, \ldots, x_{n}\right\}$, and let $\alpha$ be the nonzero rational such that $\phi(y)=\alpha \cdot y$. But then, $C$ is invertible, and $\alpha \cdot A^{\prime}=C A C^{\dagger}$, i.e., $Q_{M} \sim Q_{M^{\prime}}$. Conversely, if $Q_{M} \sim Q_{M^{\prime}}$, then there is an invertible matrix $C=\left(c_{i j}\right)$ and a nonzero rational $\alpha$ such that $\alpha \cdot A^{\prime}=C A C^{\dagger}$. Define a map $\phi: M_{n} \rightarrow M_{n}^{\prime}$ by $\phi\left(x_{i}\right)=\sum_{j=1}^{n} c_{i j} x_{j}$ for $1 \leq i \leq n$, and $\phi(y)=\alpha \cdot y$. Clearly it is an $H_{2 k-1}$-surjection, and a routine verification shows that $\phi$ is a dgL-morphism, and thus $M_{n} \ll M_{n}^{\prime}$. Since $C$ is invertible and $\alpha \neq 0$, one can construct from $C^{-1}$ and $\alpha^{-1}$ an $H_{2 k-1^{-}}$ surjection in the other direction using the same scheme, i.e., $M_{n}^{\prime} \ll M_{n}$.

Clearly, from the proof of Proposition 6 , we deduce that $\mathbb{M}$ is also classified by the set of isomorphism classes of anisotropic models, i.e., by the set of homotopy types of spaces having anisotropic models as Lie models.

Theorem 7. There is a natural semigroup isomorphism between $\overline{W(\mathbb{Q})}$ and $\mathbb{M}$.

Proof. The only thing left to do is to define the multiplication on $\mathbb{M}$. Because of Proposition 6 the following binary operation is well defined on $\mathbb{M}$. Given two anisotropic models $M_{n}=\left(\mathbb{L}(X \oplus \mathbb{Q} y), \partial y=Q_{M}\right)$ and $M_{m}^{\prime}=\left(\mathbb{L}\left(X^{\prime} \oplus \mathbb{Q} y\right), \partial y=\right.$ $Q_{M^{\prime}}$ ) one defines the operation $M_{n} \otimes_{o} M_{m}^{\prime}$ by

$$
M_{n} \otimes_{o} M_{m}^{\prime}=\left(\mathbb{L}\left(\left(X \otimes X^{\prime}\right)_{o} \oplus \mathbb{Q} y\right), \partial y=\left(Q_{M} \otimes Q_{M^{\prime}}\right)_{o}\right) .
$$

Note that we have to apply the appropriate reduction to the model $M_{n} \otimes M_{m}^{\prime}$ since it is not in general anisotropic. But the reduction process is well defined because of the Witt Decomposition Theorem. The dimension of the rational vector space $\left(X \otimes X^{\prime}\right)_{o}$ is, in general, less than the dimension of $X \otimes X^{\prime}$ and corresponds to the dimension of the reduced quadratic form $\left(Q_{M} \otimes Q_{M^{\prime}}\right)_{o}$. Finally, the Quillen model of $X_{k}=S^{2 k} \cup_{[\iota, \iota]} e^{4 k}$, i.e., $(\mathbb{L}(x, y), \partial y=[x, x])$, for which the corresponding quadratic form is $\langle 1\rangle$, clearly acts as the unity with respect to this product.

Figure 1 summarizes the results above.

\section{EXAmples}

Example 1. (Unreduced tensor product) Let $M_{n}=\left(\mathbb{L}\left(x_{1}, \ldots, x_{n}, y\right), \partial y=Q_{M}\right)$ and $M_{m}^{\prime}=\left(\mathbb{L}\left(x_{1}^{\prime}, \ldots, x_{m}^{\prime}, y^{\prime}\right), \partial^{\prime} y^{\prime}=Q_{M^{\prime}}\right)$ be two anisotropic models. Then

$$
M_{n} \otimes M_{m}^{\prime} \ll M_{n} \quad \text { and } \quad M_{m}^{\prime} .
$$

Since the quadratic forms are (regular) anisotropic, there are diagonal representations $\left\langle 1, \alpha_{2}, \ldots, \alpha_{n}\right\rangle$ and $\left\langle 1, \beta_{2}, \ldots, \beta_{m}\right\rangle$ of $Q_{M}$ and $Q_{M^{\prime}}$, respectively, up to similarity. Now

$$
Q_{M} \otimes Q_{M^{\prime}} \sim\left\langle 1, \beta_{2}, \ldots, \beta_{m}, \alpha_{2}, \alpha_{2} \beta_{2}, \ldots, \alpha_{2} \beta_{m}, \ldots \ldots, \alpha_{n}, \alpha_{n} \beta_{2}, \ldots, \alpha_{n} \beta_{m}\right\rangle .
$$




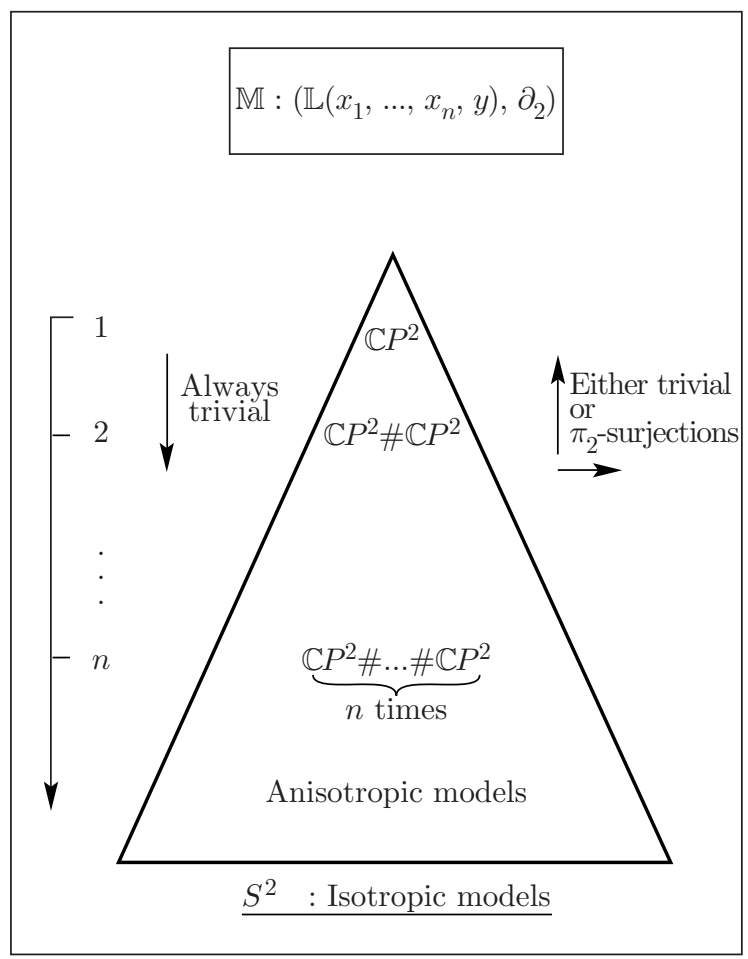

FiguRE 1.

Clearly the required $H_{2 k-1}$-surjections exist, and the statement follows. Moreover, we have

$$
M_{n} \otimes M_{m}^{\prime} \tilde{c} M_{m}^{\prime} \otimes M_{n},
$$

since $Q_{M} \otimes Q_{M^{\prime}} \sim Q_{M^{\prime}} \otimes Q_{M}$ (a simple permutation does the trick). Thus $\overline{W(\mathbb{Q})}$ and $\mathbb{M}$ are abelian semigroups.

Note that we did not apply the reduction to the resulting model. In general there is no building relationship between the two original models and their reduced product. Consider the two anisotropic models $M_{3}=\left(\mathbb{L}\left(x_{1}, x_{2}, x_{3}, y\right), \partial y=\left[x_{1}, x_{1}\right]+\right.$ $\left.\left[x_{2}, x_{2}\right]+\left[x_{3}, x_{3}\right]\right)$ and $M_{3}^{\prime}=\left(\mathbb{L}\left(x_{1}, x_{2}, x_{3}, y\right), \partial y=\left[x_{1}, x_{1}\right]+\left[x_{2}, x_{2}\right]-3\left[x_{3}, x_{3}\right]\right)$. Then the model of $M_{3} \otimes M_{3}^{\prime}$ has nine generators in degree $2 k-1$ together with

$$
\partial y \sim\langle 1,1,1,1,1,1,-3,-3,-3\rangle,
$$

which is isotropic. The reduced product is

$$
\left(\mathbb{L}\left(x_{1}, x_{2}, x_{3}, y\right), \partial y=2\left[x_{1}, x_{1}\right]+2\left[x_{2}, x_{2}\right]+3\left[x_{3}, x_{3}\right]\right) .
$$

Clearly no building relations exist among $M_{3}, M_{3}^{\prime}$, and their reduced product.

Example 2 (The Félix example). Félix showed by an argument involving the rational cohomology algebra that

$$
S^{2} \ll \ldots \ll \underbrace{\mathbb{C} P^{2} \# \ldots \# \mathbb{C} P^{2}}_{n \text { times }} \ll \ldots \ll \mathbb{C} P^{2} \# \mathbb{C} P^{2} \ll \mathbb{C} P^{2} .
$$


We can also obtain his result using Lie models. Consider

$$
\mathcal{M}(\underbrace{\mathbb{C} P^{2} \# \ldots \# \mathbb{C} P^{2}}_{n \text { times }}) \cong\left(\mathbb{L}\left(x_{1}, \ldots, x_{n}, y\right), \partial\right)
$$

where $\partial(y)=\left[x_{1}, x_{1}\right]+\cdots+\left[x_{n}, x_{n}\right], \partial\left(x_{i}\right)=0,\left|x_{i}\right|=1$, and $|y|=3$ for $1 \leq i \leq n$. The projections give the required $H_{1}$-surjections, while Lemma 5 makes it strict. Notice that the associated quadratic form is anisotropic.

Example 3. As we have seen in section 2, the sum $\perp$ is not well defined up to similarity. Thus one cannot define such an operation on the isomorphism classes of models of the type $M_{n}$ as we did for $\otimes$. Geometrically, this reflects the fact that one has to choose an orientation before making the connected sum. Clearly, homotopy invariants are blind to this choice. But given two explicit models $M_{n}=$ $\left(\mathbb{L}\left(x_{1}, \ldots, x_{n}, y\right), \partial y=Q_{M}\right)$ and $M_{m}^{\prime}=\left(\mathbb{L}\left(x_{1}^{\prime}, \ldots, x_{m}^{\prime}, y^{\prime}\right), \partial^{\prime} y^{\prime}=Q_{M^{\prime}}\right)$, one can define the (formal) connected sum as

$$
M_{n} \# M_{m}^{\prime}=\left(\mathbb{L}\left(x_{1}, \ldots, x_{n}, x_{1}^{\prime}, \ldots, x_{m}^{\prime}, y\right), \partial y=Q_{M} \perp Q_{M^{\prime}}\right) .
$$

This operation makes sense only on the (entire) set of models of the type $M_{n}$. Moreover, without lost of generality, one can assume that both $Q_{M}$ and $Q_{M^{\prime}}$ are diagonal since $\perp$ is well defined on the isometry classes. It is now clear that

$$
M_{n} \# M_{m}^{\prime} \ll M_{n} \text { and } M_{m}^{\prime}
$$

There are no simple building relations between the two operations \# and $\otimes$ as the following examples show. Let $M_{1}=(\mathbb{L}(x, y), \partial y=[x, x])$. Then

$$
M_{1} \# M_{1} \ll M_{1} \otimes M_{1} \cong M_{1},
$$

which is strict. On the other hand, we have

$$
\left(M_{1} \# M_{1} \# M_{1}\right) \otimes\left(M_{1} \# M_{1}\right) \ll\left(M_{1} \# M_{1} \# M_{1}\right) \#\left(M_{1} \# M_{1}\right),
$$

which is strict. Moreover, if $M_{2}=\left(\mathbb{L}\left(x_{1}, x_{2}, y\right), \partial y=\left[x_{1}, x_{1}\right]+2\left[x_{2}, x_{2}\right]\right)$, then the two models

$$
\left(M_{1} \# M_{1}\right) \# M_{2} \text { and }\left(M_{1} \# M_{1}\right) \otimes M_{2}
$$

are not even comparable.

Again there is no building compatibility between the original models and their reduced sum. Consider the two anisotropic models $M_{2}^{\prime}=\left(\mathbb{L}\left(x_{1}, x_{2}, y\right), \partial y=\right.$ $\left.\left[x_{1}, x_{1}\right]+\left[x_{2}, x_{2}\right]\right)$ and $M_{2}^{\prime \prime}=\left(\mathbb{L}\left(x_{1}, x_{2}, y\right), \partial y=-\left[x_{1}, x_{1}\right]+2\left[x_{2}, x_{2}\right]\right)$. Then

$$
M_{2}^{\prime} \# M_{2}^{\prime \prime}=\left(\mathbb{L}\left(x_{1}, x_{2}, x_{3}, x_{4}, y\right), \partial y=\left[x_{1}, x_{1}\right]+\left[x_{2}, x_{2}\right]-\left[x_{3}, x_{3}\right]+2\left[x_{4}, x_{4}\right]\right),
$$

which is isotropic. The reduced sum is

$$
\left(\mathbb{L}\left(x_{1}, x_{2}, y\right), \partial y=\left[x_{1}, x_{1}\right]+2\left[x_{2}, x_{2}\right]\right) .
$$

Clearly no building relations exist among $M_{2}^{\prime}, M_{2}^{\prime \prime}$, and their reduced sum. 


\section{FinAL REMARKS AND OPEN PROBLEMS}

5.1. Minimal number of generators. We have seen that one of the invariants that classify $\mathbb{M}$ is the dimension of the quadratic form. Given two anisotropic models $M_{n}=\left(\mathbb{L}\left(x_{1}, \ldots, x_{n}, y\right), \partial y=Q_{M}\right)$ and $M_{m}^{\prime}=\left(\mathbb{L}\left(x_{1}^{\prime}, \ldots, x_{m}^{\prime}, y^{\prime}\right), \partial^{\prime} y^{\prime}=Q_{M^{\prime}}\right)$ such that neither builds the other, one could ask what is the smallest number of $w_{i}$ in a model of the type $M_{p}^{\prime \prime}=\left(\mathbb{L}\left(w_{1}, \ldots, w_{p}, y\right), \partial y=Q_{M^{\prime \prime}}\right)$ that builds $M_{n}$ and $M_{m}^{\prime}$. We have seen that $p=n \cdot m$ for $M_{n} \otimes M_{m}^{\prime}$, while $p=n+m$ for $M_{n} \# M_{m}^{\prime}$. One could do it with one less, i.e., if $Q_{M}=\left\langle 1, \alpha_{2}, \ldots, \alpha_{n}\right\rangle$ and $Q_{M^{\prime}}=\left\langle 1, \beta_{2}, \ldots, \beta_{m}\right\rangle$, then we can set $p=n+m-1$ together with

$$
Q_{M^{\prime \prime}}=\left\langle 1, \alpha_{2}, \ldots, \alpha_{n}, \beta_{2}, \ldots, \beta_{m}\right\rangle .
$$

It is still unknown if one could do better in general. Note that both $M_{n} \otimes M_{m}^{\prime}$ and $M_{n} \# M_{m}^{\prime}$ build $M_{p}^{\prime \prime}$.

5.2. Geometric interpretation of $M_{n} \otimes M_{m}^{\prime}$ (unreduced). If one allows for a shift in the degrees of the generators, then the smash product $M_{n} \wedge M_{m}^{\prime}$ has all the required characteristics of $M_{n} \otimes M_{m}^{\prime}$. Let

$$
M_{n}=\left(\mathbb{L}\left(x_{1}, \ldots, x_{n}, y\right), \partial y=Q_{M}\right) \text { and } M_{m}^{\prime}=\left(\mathbb{L}\left(x_{1}^{\prime}, \ldots, x_{m}^{\prime}, y^{\prime}\right), \partial^{\prime} y^{\prime}=Q_{M^{\prime}}\right)
$$

be two models. Recall that the smash product is the homotopy cofibre of the following inclusion:

$$
M_{n} \vee M_{m}^{\prime} \hookrightarrow M_{n} \times M_{m}^{\prime}
$$

Let $\partial y=\sum_{i=1}^{n} \alpha_{i}\left[x_{i}, x_{i}\right]$ and $\partial^{\prime} y^{\prime}=\sum_{j=1}^{m} \beta_{j}\left[x_{j}^{\prime}, x_{j}^{\prime}\right]$ be two diagonal representations of $Q_{M}$ and $Q_{M^{\prime}}$, respectively. A model for the product is given by (see [18])

$$
\left(\mathbb{L}\left(x_{i}, x_{j}^{\prime}, c_{i j}, y, y^{\prime}, d_{i}, e_{j}, z\right), \delta\right),
$$

where $1 \leq i \leq n, 1 \leq j \leq m,\left|c_{i j}\right|=4 k-1,\left|d_{i}\right|=\left|e_{j}\right|=6 k-1$ and $|z|=8 k-1$. Moreover, after a simple computation ensuring that $\delta^{2}=0$ we get

1. $\delta x_{i}=\delta x_{j}^{\prime}=0$;

2. $\delta y=Q_{M}$, and $\delta y^{\prime}=Q_{M^{\prime}}$;

3. $\delta c_{i j}=\left[x_{i}, x_{j}^{\prime}\right]$;

4. $\delta d_{i}=\left[x_{i}, y^{\prime}\right]-2 \sum_{j=1}^{m} \beta_{j}\left[x_{j}^{\prime}, c_{i j}\right]$;

5. $\delta e_{j}=\left[y, x_{j}^{\prime}\right]+2 \sum_{i=1}^{n} \alpha_{i}\left[c_{i j}, x_{i}\right] ;$ and

6.

$$
\begin{aligned}
\delta z=\left[y, y^{\prime}\right] & -2\left(\sum_{i=1}^{n} \alpha_{i}\left[d_{i}, x_{i}\right]+\sum_{j=1}^{m} \beta_{j}\left[x_{j}^{\prime}, e_{j}\right]\right) \\
& +2 \sum_{i=1}^{n} \sum_{j=1}^{m} \alpha_{i} \beta_{j}\left[c_{i j}, c_{i j}\right] .
\end{aligned}
$$

Thus $M_{n} \wedge M_{m}^{\prime}$ has as Lie model $\left(\mathbb{L}\left(c_{i j}, d_{i}, e_{j}, z\right), \bar{\delta}\right)$, where

$$
\bar{\delta} z=2 \sum_{i=1}^{n} \sum_{j=1}^{m} \alpha_{i} \beta_{j}\left[c_{i j}, c_{i j}\right]
$$


is the only nontrivial differential. Clearly this quadratic form is similar to $Q_{M} \otimes$ $Q_{M^{\prime}}$. From the initial remarks of section 3 , we obviously have

$$
M_{n} \wedge M_{m}^{\prime} \tilde{c}\left(\mathbb{L}\left(c_{i j}, z\right), \bar{\delta}\right),
$$

i.e., we fall back on a model of $M_{n} \otimes M_{m}^{\prime}$ modulo the aforementioned shift in the degrees of the generators.

Finally, notice that the fact that $Q_{M} \otimes Q_{M^{\prime}}$ is well defined up to similarity shows that $M_{n} \wedge M_{m}^{\prime}$ is independent of the chosen homotopy representative for $M_{n}$ and $M_{m}^{\prime}$, respectively, a fact that does not hold for the connected sum.

5.3. Models $\left(\mathbb{L}(X \oplus Y), \partial=\partial_{2}\right)$ with $\operatorname{dim}(Y)=n>1$. This case, in which each model can be identified with a system of $n$ quadratic forms, is far from being classified. As mentioned in [14, Chapter 9, there is no natural way of defining a Witt group on such systems. Nonetheless, one can define the notion of an isotropic system if that system has a common nontrivial zero. Proposition 4 generalizes in this context, but Lemma 5 has no such generalization, as the following examples show. Let $M_{1}=(\mathbb{L}(x, y), \partial y=[x, x]), M_{2}=\left(\mathbb{L}\left(x_{1}, x_{2}, y\right), \partial y=\left[x_{1}, x_{1}\right]+\left[x_{2}, x_{2}\right]\right)$, and $M_{2}^{\prime}=\left(\mathbb{L}\left(x_{1}, x_{2}, y\right), \partial y=\left[x_{1}, x_{1}\right]+2\left[x_{2}, x_{2}\right]\right)$, and consider the spaces

$$
M_{1} \vee M_{1} \cong\left(\mathbb{L}\left(x_{1}, x_{2}, y_{1}, y_{2}\right), \partial y_{1}=\left[x_{1}, x_{1}\right], \partial y_{2}=\left[x_{2}, x_{2}\right]\right),
$$

and

$$
\begin{aligned}
M_{2} \vee M_{2}^{\prime} \cong\left(\mathbb{L}\left(x_{1}, x_{2}, x_{3}, x_{4}, y_{1}, y_{2}\right), \partial y_{1}\right. & =\left[x_{1}, x_{1}\right]+\left[x_{2}, x_{2}\right], \\
\partial y_{2} & \left.=\left[x_{3}, x_{3}\right]+2\left[x_{4}, x_{4}\right]\right) .
\end{aligned}
$$

Clearly the inclusion $M_{1} \hookrightarrow M_{1} \vee M_{1}$ into either the left or right component is nontrivial, while the projection of $M_{2} \vee M_{2}^{\prime}$ onto either $M_{2}$ or $M_{2}^{\prime}$ is also nontrivial.

Finally, all is not lost, since there is an obvious action of $\mathbb{M}$ on the set of such models. Namely, given two models $M=(\mathbb{L}(X \oplus \mathbb{Q} y), \partial y=Q)$ and $N=\left(\mathbb{L}\left(X^{\prime} \oplus\right.\right.$ $\left.\left.\mathbb{Q} y_{1} \oplus \ldots \oplus \mathbb{Q} y_{n}\right), \partial y_{1}=Q_{1}, \ldots, \partial y_{n}=Q_{n}\right)$ we have

$$
M \cdot N=\left(\mathbb{L}\left(X \otimes X^{\prime} \oplus \mathbb{Q} y_{1} \oplus \ldots \oplus \mathbb{Q} y_{n}\right), \partial y_{1}=Q \otimes Q_{1}, \ldots, \partial y_{n}=Q \otimes Q_{n}\right) .
$$

Note that we cannot hope to diagonalize simultaneously a system of quadratic forms. Thus the product is the Kronecker product of two matrices.

\section{Appendix : Classification of similarity Classes}

We have to distinguish the odd-dimensional case from the even-dimensional one. The odd case is easily disposed of.

Proposition 8. Let $Q_{1}$ and $Q_{2}$ be two $(2 k+1)$-dimensional regular quadratic forms. Then

$$
Q_{1} \sim Q_{2} \quad \text { if and only if }\left(\operatorname{det}\left(Q_{1}\right)\right) Q_{1} \simeq\left(\operatorname{det}\left(Q_{2}\right)\right) Q_{2} .
$$

Proof. Suppose we have $r \cdot Q_{1} \simeq Q_{2}$, i.e., there exists an invertible matrix $C$ such that $r \cdot Q_{1}=C Q_{2} C^{\dagger}$. Applying the determinant to each side yields

$$
r^{(2 k+1)} \cdot \operatorname{det}\left(Q_{1}\right)=(\operatorname{det}(C))^{2} \cdot \operatorname{det}\left(Q_{2}\right) .
$$

Thus $r \equiv\left(\operatorname{det}\left(Q_{1}\right) \cdot \operatorname{det}\left(Q_{2}\right)\right)$ modulo $\left(\mathbb{Q}^{*}\right)^{2}$. The result follows.

The similarity classes of even-dimensional quadratic forms have only recently been classified, by Lewis and Tignol. 
Proposition 9 ([13)). Let $Q_{1}$ and $Q_{2}$ be two even-dimensional regular quadratic forms. Then $Q_{1} \sim Q_{2}$ if and only if

- $\operatorname{dim}\left(Q_{1}\right)=\operatorname{dim}\left(Q_{2}\right)$;

- $\operatorname{det}\left(Q_{1}\right) \equiv \operatorname{det}\left(Q_{2}\right)$ modulo $\left(\mathbb{Q}^{*}\right)^{2}$;

- $C_{o}\left(Q_{1}\right) \cong C_{o}\left(Q_{2}\right)$ as algebras over $\mathbb{Q}$; and

- $\left|\operatorname{sign}\left(Q_{1}\right)\right|=\left|\operatorname{sign}\left(Q_{2}\right)\right|$.

Recall that $C_{o}(Q)$ refers to the even Clifford algebra associated to $Q$ (see [16]).

\section{ACKNOWLEDGMENT}

The second author would like to thank colleagues at l'École Polytechnique Fédérale de Lausanne for their hospitality and generosity. These two weeks of March 2001 were instrumental in getting this project up and running. We also want to thank Y. Félix and J.-P. Tignol for the very enlightening discussions we had with them. Finally, we thank D. Stanley for suggesting the geometric interpretation of $M_{n} \otimes M_{m}^{\prime}$.

\section{REFERENCES}

[1] J.F. Adams, P.J. Hilton, On the chain algebra of a loop space, Comment. Math. Helvetici 30, (1956), pp. 305-330. MR 17:1119b

[2] D.J. Anick, Hopf algebras up to homotopy, J. Amer. Math. Soc. 2, (1989), pp. 417-453. MR 90c: 16007

[3] A.K. Bousfield, Localization and periodicity in unstable homotopy theory, J. Amer. Math. Soc. 7, No.4 (1994), pp. 831-874. MR 95c:55010

[4] A.K. Bousfield, D.M. Kan, Homotopy Limits, Completions, and Localizations, Springer Lecture Notes in Math. 304, (1972). MR 51:1825

[5] W. Chachólski, Closed classes, Proc. Conf. on Algebraic Topology, Barcelona (1994), Progress in Math. Vol. 136, Birkhäuser (1996), pp. 95-118. MR 97e:55007]

[6] W. Chachólski, P.-E. Parent, and D. Stanley, A-acyclic spaces versus A-cellular spaces, preprint.

[7] W. Chachólski, P.-E. Parent, and D. Stanley, Cellular generators, Proc. Amer. Math. Soc., To appear.

[8] E. D. Farjoun, Cellular Spaces, Null Spaces and Homotopy Localization, Lecture Notes in Mathematics 1622 (1996). MR 98f:55010

[9] Y. Félix, S. Halperin, and J.-C. Thomas, Rational homotopy theory, Graduate Texts in Mathematics 205, Springer-Verlag, (2001). MR 2002d:55014

[10] S. Halperin, J.-M. Lemaire, Suites inertes dans les algèbres de Lie graduées, Math. Scand. 61, (1987), pp. 39-67. MR 89e:55022

[11] K. Hess, The rational homotopy algebra and cellular type, preprint.

[12] M.J. Hopkins, J.H. Smith, Nilpotence and stable homotopy theory II, Ann. of Math. 148, (1998), pp. 1-49. MR 99h:55009

[13] D.W. Lewis, J.-P. Tignol, Classification theorems for central simple algebras with involution, Manuscripta Math. 100, (1999), pp. 259-276. MR 2000m:16027

[14] A. Pfister, Quadratic forms with applications to algebraic geometry and topology, London Math. Soc., Lecture Note Series 217, Cambridge University Press, (1995). MR 97c:11046

[15] D. Quillen, Rational homotopy theory, Annals of Math. (2) 90, (1969), pp. 205-295. MR 41:2678

[16] W. Scharlau, Quadratic and hermitian forms, A series of comprehensive studies in mathematics 270, Springer-Verlag, (1985). MR 86k:11022 
[17] D. Sullivan, Infinitesimal computations in topology, Inst. Hautes Études Sci. Publ. Math. 47 (1977), pp. 269-331. MR 58:31119]

[18] D. Tanré, Homotopie Rationnelle: Modèles de Chen, Quillen, Sullivan, Lecture Notes in Math. 1025, Springer-Verlag, (1983). MR 86b:55010

Chaire d'Algèbre, Département de Mathématiques, Ecole Polytechnique Fédérale, 1015 Lausanne, Suisse

E-mail address: kathryn.hess@epfl.ch

Chaire d'Algèbre, Département de Mathématiques, Ecole Polytechnique Fédérale, 1015 Lausanne, Suisse

E-mail address: paul-eugene.parent@epfl.ch 